\title{
The Application of X-STR: Two Case Reports
}

\author{
Zhengliang $\mathrm{Yu}^{1}$, Jing Sun ${ }^{1}$, Guangshu Ding ${ }^{1}$, Xi Wang ${ }^{1}$, Hong $\mathrm{Liu}^{2}$, Xingchun Zhao ${ }^{1}$ and Wanshui $\mathrm{Li}^{1}$ \\ 1. Institute of Forensic Science, Ministry of Public Security, Beijing 100038, China \\ 2. Guangzhou Forensic Institute, Guangzhou 510000, China
}

\begin{abstract}
The usefulness of the X-chromosomal STRs (short tandem repeats) for forensic purposes seems to be restricted, but may be valuable in some paternity cases. Due to the particular mode of inheritance, X-chromosomal X-STR has the advantage in female traces identification against male contamination and in complex kinship cases, such as deficiency cases (mother-son or father-daughter), grandparent-grandchild comparisons, half-sisters testing, etc. In this study, 4 blood samples from two paternity cases without father were examined with TYPER19 Amplification kit first, from the result, it is hard to judge the relationship between the mother-sons, then TYPERX19 kit was conducted to obtain the full profiles of the two pairs of the mother-son, from the genetypes of the samples, it can be inferred that neither of the two pairs of mother-son were in line with the kinship, which provides important evidence for the two paternity cases. All together, the X-chromosome marks included in the TYPERX19 kit can offer the possibility to solve complex kinship cases where autosomal STR markers cannot provide the information needed.
\end{abstract}

Key words: X-STR, identification, kinship.

\section{Introduction}

Paternity trio cases can most easily be solved with autosomal STR (short tandem repeat) markers alone, Y-chromosomal STRs can provide clues for male pedigree investigation, so most forensic interests have been focused on autosomal and Y-chromosomal STRs, while test of paternity duos involving a daughter or more complex family relations could gain from $\mathrm{X}$-chromosomal testing, analysis of X-STR can be beneficial in identification on a trace with female minor component and male major component and in complex kinship cases, such as deficiency cases (mother-son or father-daughter), grandparent-grandchild comparisons, half-sisters testing, paternity testing in incest cases and so forth [1]. TYPERX19 kit is one of the most commonly used kits for X-STR typing, which includes 18 X-STR loci (DXSGATA31E08, DXS10079, DXS10103, DXS7132, DXS9895, DXS7133, DXS7424, DXS7423, DXS6789, DXS9902, DXS6810, DXS8377, DXS101, HPRTB, DXS8378, DXS6797, DXS6804, GATA165B12) and one amelogenin loci.

Corresponding author: Wanshui Li, bachelor, professor, research fields: forensic genetics.
$\mathrm{X}$-STRs have been recognized as important tools in forensic application [2-11]. Especially in some identification of kinship, the test efficiency can be significantly improved by adding the X-STR test when no conclusion can be drawn by the autosomal STR test [12-15]. Therefore, X-STR can serve as an important complement to autosomal STR, Y-STR and mitochondrial DNA [16]. This study introduced two paternity cases, in which X-STR played an important role.

\section{Materials and Methods}

Four blood samples from two paternity cases without father were collected from Institute of Forensic Science, Ministry of Public Security, Beijing, China. These two pairs of mother-son were from Sichuan province, the child needs to do kinship identification before registering their household registration. The samples were amplified with TYPER19 and TYPERX19 Amplification kit following manufacturer's recommendations. Gene-Mapper ID-X v1.3 (Life Technologies, USA) was used to determine fragment size and genotyping. Allele peak of 100 relative fluorescence unit (RFU) was implemented as the peak 
detection threshold when analyzing data from CE instruments.

\section{Results and Discussion}

In these two paternity cases, the samples were amplified with TYPER19 (18 autosomal STR and one Amelogenin loci) first, the genotyping of the son is mismatched with that of the mother at D16S539 loci in case 1 (see Table 1), the genotyping of the son is mismatched with that of the mother at D8S1179 and D19S433 loci in case 2 (see Table 2). The mismatch can be probably due to the mutation in these two cases, it is difficult to judge their mother-son relationship, then these four samples were amplified with the TYPERX19, the genotyping was shown in Tables 3 and 4, there were $8 \mathrm{X}$-STRs that were mismatched in case 1 , there were 4 $\mathrm{X}$-STRs that were mismatched in case 2, according to the number of inconsistent loci and the mutation rate of X-STR [17-20], it can be judged that neither of the two pairs of mother-son were in line with the kinship, in the subsequent investigation of the mother-sons, it was confirmed that two pairs of the mother-son were both non-biological, it verified our results. X-STR played an important role in these two cases.

Table 1 The gene-type of the samples in case 1 (amplified with TYPER19 Amplification kit).

\begin{tabular}{llllllll}
\hline & AMEL & D5S818 & D21S11 & D7S820 & CSF1PO & D2S1338 & D3S1358 \\
\hline $5392-1$ & X & 9,11 & $30,30.3$ & 11,12 & 12,14 & 22,23 & 16 \\
$5392-2$ & X,Y & 9,11 & $29.2,30$ & 11 & 13,14 & 18,23 & 16,17 \\
& vWA & D8S1179 & D16S539 & Penta E & TPOX & TH01 & D19S433 \\
$5392-1$ & 17,18 & 14,15 & 11,12 & 5,13 & 8 & 7 & $14,14.2$ \\
$5392-2$ & 17 & 14,15 & 9,13 & 13 & 8,11 & 7,9 & $13,14.2$ \\
& D18S51 & FGA & D6S1043 & D13S317 & D12S391 & & \\
$5392-1$ & 13,15 & 18,24 & 13,19 & 10,11 & 16,17 & & \\
$5392-2$ & 13,14 & 24,26 & 19 & 10,12 & 17 & & \\
\hline
\end{tabular}

Table 2 The gene-type of the samples in case 2 (amplified with TYPER19 Amplification kit).

\begin{tabular}{llllllll}
\hline & AMEL & D5S818 & D21S11 & D7S820 & CSF1PO & D2S1338 & D3S1358 \\
\hline $3753-1$ & X & 11,12 & $29.2,31.2$ & 10,12 & 12 & 21,23 & 15,16 \\
$3753-2$ & X,Y & 11,15 & $29.2,31.2$ & 8,10 & 12 & 20,23 & 15 \\
& vWA & D8S1179 & D16S539 & Penta E & TPOX & TH01 & D19S433 \\
$3753-1$ & 16,18 & 12,16 & 11 & 5,12 & 8,11 & 7 & $14.2,15.2$ \\
$3753-2$ & 18,19 & 10,13 & 10,11 & 12,19 & 8 & 7,9 & 14,15 \\
& D18S51 & FGA & D6S1043 & D13S317 & D12S391 & & \\
$3753-1$ & 15,16 & 23,24 & 14,18 & 9 & 18,20 & & \\
$3753-2$ & 15,22 & 21,23 & 18 & 9,11 & 18,19 & & \\
\hline
\end{tabular}

Table 3 The gene-type of the samples in case 1 (amplified with TYPERX19 Amplification kit).

\begin{tabular}{llllllll}
\hline & AMEL & GATA31E08 & DXS10079 & DXS10103 & DXS7132 & DXS9895 & DXS7133 \\
\hline $5392-1$ & X & 9,12 & 18,22 & 16,19 & 12,14 & 15,16 & 9 \\
$5392-2$ & X,Y & 11 & 19 & 16 & 13 & 16 & 9 \\
& DXS7424 & DXS7423 & DXS6789 & DXS9902 & DXS6810 & DXS8377 & DXS101 \\
$5392-1$ & 14,16 & 15 & 16,20 & 10 & 17,18 & 49 & 24,25 \\
$5392-2$ & 16 & 15 & 19 & 10 & 18 & 49 & 27 \\
& HPRTB & DXS8378 & DXS6797 & DXS6804 & GATA165B12 & & \\
$5392-1$ & 13 & 10 & 23 & 12,14 & 9,11 & & \\
$5392-2$ & 12 & 10 & 24 & 9 & 11 & & \\
\hline
\end{tabular}


Table 4 The gene-type of the samples in case 2 (amplified with TYPERX19 Amplification kit).

\begin{tabular}{llllllll}
\hline & AMEL & GATA31E08 & DXS10079 & DXS10103 & DXS7132 & DXS9895 & DXS7133 \\
\hline $3753-1$ & X & 10 & 18,21 & 16 & 11,13 & 13,14 & 9 \\
$3753-2$ & X,Y & 10 & 20 & 16 & 13 & 17 & 9 \\
& DXS7424 & DXS7423 & DXS6789 & DXS9902 & DXS6810 & DXS8377 & DXS101 \\
$3753-1$ & 15,16 & 15 & 16,19 & 10 & 18,19 & 43,48 & 24,25 \\
$3753-2$ & 15 & 15 & 16 & 11 & 18 & 48 & 25 \\
& HPRTB & DXS8378 & DXS6797 & DXS6804 & GATA165B12 & & \\
$3753-1$ & 12,14 & 11 & 24 & 10,12 & 10,11 & & \\
$3753-2$ & 14 & 12 & 24 & 12 & 11 & & \\
\hline
\end{tabular}

\section{Conclusions}

In the identification of deficiency cases (mother-son or father-daughter), grandparent-grandchild comparisons, half-sisters testing, etc., where autosomal STR markers cannot provide the information needed, $\mathrm{X}$-STR can be added for testing, which can play an important role in these cases.

\section{Conflict of interest}

The authors declare that there is no conflict of interest with regard to publication of this article. The institute, from where the data for this study were collected, has been duly acknowledged in the material and methods section.

\section{Funding}

This work was supported by a grant from Technology Research Program of Ministry of Public Security (Grant No. 2016JSYJC14), a grant from Public Research Program of China Institute of Forensic Science (Grant No. 2016CSEEKFKT03) and a grant from Fundamental research funds of China Institute of Forensic Science, Ministry of Public Security (Grant No. 2018JB024).

\section{References}

[1] Szibor, R. 2007. "X-Chromosomal Markers: Past, Present and Future.” Forensic Sci. Int. Genet. 1: 93-9.

[2] Eroğlu, A., Yüksel, B., Ergüder, B. I., et al. 2015. "Importance of X-STR Analysis in Forensic Science." Journal of Forensic Medicine 29 (3): 187-94.

[3] Szibor, R., Krawczak, M., Hering, S., et al. 2003. "Use of X-Linked Markers for Forensic Purposes.” Int J Legal Med. 117: 67-74.
[4] Diegoli, T. M. 2015. "Forensic Typing of Short Tandem Repeat Markers on the X and Y Chromosomes.” Forensic Sci. Int. Genet. 18: 140-51.

[5] Nothnagel, M., Szibor, R., Vollrath, O., et al. 2012. "Collaborative Genetic Mapping of 12 Forensic Short Tandem Repeat (STR) Loci on the Human X Chromosome." Forensic Science International: Genetics 6 (6): 778-84.

[6] Edelmann, J., and Szibor, R. 2001. "DXS101: A Highly Polymorphic X-Linked STR.” Int. J. Legal Med. 114: 301-4.

[7] Szibor, R., Lautsch, S., Plate, I., and Beck, N. 2000. "Population Data on the X Chromosome Short Tandem Repeat Locus HumHPRTB in Two Regions of Germany.” J. Forensic Sci. 45: 231-3.

[8] Edelmann, J., Hering, S., Michael, M., Lessig, R., Deichsel, D., Meier-Sundhausen, G., et al. 2001. “16 Xchromosome STR Loci Frequency Data from a German Population.” Forensic Sci. Int. 124: 215-8.

[9] Edelmann, J., Deichsel, D., Hering, S., Plate, I., and Szibor, R. 2002. "Sequence Variation and Allele Nomenclature for the X-Linked STRs DXS9895, DXS8378, DXS7132, DXS6800, DXS7133, GATA172D05, DXS7423 and DXS8377.” Forensic Sci. Int. 129: 99-103.

[10] Zarrabeitia, M. T., Amigo, T., Sañudo, C., Zarrabeitia, A., Gonza'lez-Lamuñ, D., and Riancho, J. A. 2002. “A New Pentaplex System to Study Short Tandem Repeat Markers of Forensic Interest on X Chromosome.” Forensic Sci. Int. 129: 85-9.

[11] Wiegand, P., Berger, B., Edelmann, J., Parson, W. 2003. "Population Genetic Comparisons of Three X-Chromosomal STRs.” Int. J. Legal Med. 117: 62-5.

[12] Dayse, A. S., Femanda, S. N. M, Marcia, D., et al. 2009. "Paternity Testing Involving Human Remain Identification and Putative Half Sister: Usefulness of an X-Hexaplex STR Markers.” Forensic Science International: Genetics Supplement Series 2: 230-1.

[13] Chiara, T., Silvano, P., Isabella, S., et al. 2006. "Usefulness of X-Chromosome Markers in Resolving Relationships: Report of a-Court Case Involving Presumed Half Sisters.” International Congress Series 
1288: 301-3.

[14] Wang, X. X., Chen, M., Li, R. M., et al. 2014. "Identification of the Relationship of Orphaned Sisters by Combined Using Autosomal STR, X-STR and Mitochondrial-SNP.” Journal of Molecular Diagnostics and Therapy 6: 12-7.

[15] Pinto, N., Gusmão, L., and Amorim, A. 2003. "X-Chromosome Markers in Kinship Testing: A Generalisation of the IBD Approach Identifying Situations Where Their Contribution Is Crucial." Forensic Sci. Int. Genet. 5: 27-32.

[16] Szibor, R., Krawczak, M., Hering, S., Edelmann, J., Kuhlisch, E., and Krause, D. 2003. "Use of X-Linked Markers for Forensic Purposes.” Int. J. Legal Med. 117: 67-74.

[17] Tillmar, A. O., Mostad, P., Egeland, T., Lindblom, B.,
Holmlund, G., and Montelius, K. 2008. "Analysis of Linkage and Linkage Disequilibrium for Eight X-STR Markers.” Forensic Science International: Genetics 3: 37-41.

[18] Fracasso, T., Schürenkamp, M., Brinkmann, B., and Hohoff, C. 2008. "An X-STR Meiosis Study in Kurds and Germans: Allele Frequencies and mutation Rates.” International Journal of Legal Medicine 122: 353-6.

[19] Liu, Q. L., Lu, D. J., Li, X. G., Zhao, H., Zhang, J. M., Lai, Y. K., and Chen, Y. F. 2011. "Development of the Nine X-STR Loci Typing System and Genetic Analysis in Three Nationality Populations from China.” Int. J. Legal Med. 125: 51-8.

[20] Castañeda, M., Mijares, V., Riancho, J. A., and Zarrabeitia, M. T. 2012. "Haplotypic Blocks of X-Linked STRs for Forensic Cases: Study of Recombination and Mutation Rates.” J. Forensic Sci. 57: 192-5. 